\title{
Refractory Colon Adenocarcinoma
}

National Cancer Institute

\section{Source}

National Cancer Institute. Refractory Colon Adenocarcinoma. NCI Thesaurus. Code C162155.

Colon adenocarcinoma that is resistant to treatment. 